
\title{
25 Research Soure \\ Effects of SDF-1/CXCR7 on the Migration, Invasion and Epithelial-Mesenchymal Transition of Gastric Cancer Cells
}

\author{
Ameng Shi \\ the Second Affiliated Hospital of Xi'an Jiaotong University \\ Ting Wang \\ the Second Affiliated Hospital of Xi'an Jiaotong University \\ Miao Jia \\ the Second Affiliated Hospital of Xi'an Jiaotong University \\ Lei Dong \\ the Second Affiliated Hospital of Xi'an Jiaotong University \\ Haitao Shi ( $\nabla$ shihaitao7@163.com )
}

the Second Affiliated Hospital of Xi'an Jiaotong University https://orcid.org/0000-0003-2354-5209

\section{Research Article}

Keywords: chemokine (CXC motif) receptor 7 (CXCR7), migration, invasion, epithelial-mesenchymal transition (EMT), gastric cancer

Posted Date: August 12th, 2021

DOI: https://doi.org/10.21203/rs.3.rs-767692/v1

License: (c) (1) This work is licensed under a Creative Commons Attribution 4.0 International License. Read Full License 


\section{Abstract}

We found that SDF-1/CXCR7 axis plays an important role in the growth and proliferation of gastric cancer in previous studies. The objectives of this study were to explore the effects of SDF-1/CXCR7 on the metastatic ability of gastric cancer cells and the possible mechanisms. SGC-7901 gastric cancer cells were cultured in vitro, CXCR7 expression was stably knocked down via lentiviral vectors. The cell migration and invasion abilities were detected by transwell migration and invasion assays. The expression of matrix metalloproteinase 2 (MMP-2), MMP-9, vascular endothelial growth factor (VEGF), epithelial-mesenchymal transition (EMT) markers and Akt phosphorylation were detected with western blot. We found that SDF-1 markedly enhanced the migration and invasion abilities of SGC-7901 gastric cancer cells; CXCR7 knockdown by lentiviral infection inhibited these effects. SDF-1/CXCR7 increased the expression of MMP-2, MMP-9 and VEGF. SDF-1/CXCR7 also downregulated E-cadherin expression but upregulated N-cadherin, vimentin and Snail expression, suggesting that SDF-1/CXCR7 could promote the development of EMT in gastric cancer cells. Furthermore, SDF-1/CXCR7 could promote Akt phosphorylation. Our results indicated that SDF-1/CXCR7 promoted the migration, invasion and EMT of gastric cancer cells and thus CXCR7 inhibition may be a strategy for inhibiting gastric cancer metastasis.

\section{Introduction}

According to Global Cancer Statistics 2018, gastric cancer is the fifth most common malignant tumor worldwide [1]. Surgery, chemotherapy, radiotherapy, molecular targeted therapy and immunotherapy are still the major treatment currently. Early gastric cancer is difficult to detect, as there are few clinical symptoms. However, if active treatment can be provided on the basis of early diagnosis, the 5-year survival rate can reach more than $90 \%$, with the possibility of achieving cure. The prognosis of patients with progressed gastric cancer is relatively poor. Even with surgery, the survival rate is still lower than $30 \%$ [2]. Therefore, it's still a challenge for us to study the molecular mechanisms of gastric carcinogenesis and searching for new therapeutic targets.

CXCL12, also known as stromal derived factor-1 (SDF-1), has two receptors, CXCR4 and CXCR7. Many studies have found that SDF-1/CXCR4 axis regulates gastric cancer proliferation, migration, invasion, metastasis and angiogenesis [3]. CXCR7 was discovered in recent years and lacks the classical DRYLAIV motif and cannot mediate Gai protein activation [4]. CXCR7 has significantly higher affinity than CXCR4 for SDF-1/CXCL12. CXCR7 and CXCR4 can each form homodimers and heterodimers. When CXCR7 and CXCR4 were co-expressed, both of the two receptors could be activated in the presence of SDF-1.

Therefore, the role of SDF/CXCR7 in pathophysiology has been getting more attention gradually. CXCR7 expression is upregulated in tumors; CXCR7 affects tumor growth and metastasis and is associated with poor prognosis. We have long focused on the role of CXCR7 in gastric cancer. Our previous study found that the increased expression of CXCR7 in gastric cancer tissues was correlated with tumor size and lymph node metastasis. In addition, we also found that SDF-1/CXCR7 accelerated the proliferation of SGC-7901 gastric cancer cells and the extracellular signal-regulated kinase1/2 (ERK1/2) and p38 signaling pathways might be involved these effects[5]. However, its effects on the metastasis of gastric 
cancer and relevant mechanisms especially the Epithelial-mesenchymal transition (EMT) are not clear yet. Existing researches revealed that CXCR7 can increase the in vitro migration and invasion abilities of some tumors, including liver cancer [6-8], pancreatic cancer [9], colon cancer [10], kidney cancer [11], breast cancer [12], ovarian cancer [13], prostate cancer [14, 15], bladder cancer [16], and glioma [17]. However, the related mechanism is not very clear. EMT and angiogenesis are important factors in tumor metastasis and targeting them is a potential therapeutic strategy. Therefore, the current research is mainly to explore the effects of SDF-1/CXCR7 on the migration, invasion, angiogenesis and EMT of gastric cancer cells in vitro.

\section{Materials And Methods}

\section{Cells culture}

SGC-7901 (Shanghai Institute of Cell Research, Shanghai, China), a moderately differentiated human gastric adenocarcinoma cell line, was incubated in RPMI 1640 medium (HyClone, Logan, UT, USA) supplemented with $10 \%$ fetal bovine serum (Sijiqing, Hangzhou, China) at $37^{\circ} \mathrm{C}$ in $5 \% \mathrm{CO}_{2}$ incubator.

\section{CXCR7 knockdown}

Three shRNA-expressing lentiviral vectors (LV) including LV-negative control (LV-NC), LV-CXCR7-1 and LVCXCR7-2 were from Cyagen Biosciences. CXCR7-siRNA-1 was 5'-CGCACTGCTACATCTTGAA-3', CXCR7siRNA-2 was 5'-GCCGTTCCCTTCTCCATTATC-3'. In the presence of polybrene $(5 \mu \mathrm{g} / \mathrm{ml})$, the SGC-7901 cells infected with these three kinds of LV were selected by puromycin $(1.5 \mu \mathrm{g} / \mathrm{ml})$ (Sigma-Aldrich, Saint Louis, MO, USA). Reverse transcription quantitative real-time PCR (RT-qPCR) and western blot were used to detected the mRNA and protein expression.

\section{Reverse transcription quantitative real-time PCR (RT-qPCR)}

RNA extraction kit (Fastagen, Shanghai, China) was used to extract total RNA according to the manufacturer's instruction. The RNA purity and concentration were measured with Thermo Scientific NanoDrop spectrophotometer. cDNA was synthesized according to the instructions of PrimeScriptTM RT Master Kit (TaKaRa, Otsu, Japan). The required primer sequences are listed in Table 1. SYBR Premix Ex TaqTM II kit (TaKaRa, Otsu, Japan) was used to perform RT-qPCR according to the manufacturer's protocol. The applied PCR conditions were: preliminary denaturation at $95^{\circ} \mathrm{C}$ for $30 \mathrm{~s}$, followed by 40 cycles at $95^{\circ} \mathrm{C}$ for $5 \mathrm{~s}$ and $60^{\circ} \mathrm{C}$ for $30 \mathrm{~s}$. The $2^{-\triangle \triangle C T}$ (CT: threshold cycle) method was used to calculate the RNA expression levels.

\section{Western blot}

RIPA lysis buffer (Beyotime, China) was used to extract total protein according to the manufacturer's instruction. The total protein sample was separated by SDS-PAGE, transferred to a PVDF membrane (Millipore, Billerica, MA, USA), blocked in non-fat milk, incubated with primary antibodies at suitable 
dilution concentration followed by secondary antibody. The protein bands were detected using an ECL plus chemiluminescence detection kit (Millipore, Billerica, MA, USA). Gel-pro Analyzer 4.0 software (Media Cybernetics, CA, USA) was used to analyze the integral optical density (IOD). Polyclonal antibodies against CXCR7, MMP-2, MMP-9, VEGF, E-cadherin, N-cadherin, vimentin, snail, $\beta$-actin and secondary antibody were purchased from Santa Cruz, Dallas, TX, USA. Monoclonal antibodies against p-Akt and tAkt were purchased from Cell Signaling Technology, Danvers, MA, USA.

\section{Cell migration assay}

Serum starved LV-NC and LV-CXCR7-1 cells were resuspended at a cell density of $5 \times 10^{5} \mathrm{cell} / \mathrm{s} / \mathrm{ml}$ in medium containing $1 \%$ FBS. $200 \mu \mathrm{l}$ of the cell suspension was added to the upper chamber of a 24-well transwell, and $600 \mu \mathrm{l}$ of medium containing $10 \%$ FBS with or without SDF-1 $(100 \mathrm{ng} / \mathrm{ml})$ was added into the lower chamber. There were four experimental groups: LV-NC, LV-NC+SDF-1, LV-CXCR7-1 and LVCXCR7-1+SDF-1. After $48 \mathrm{~h}$ of incubation, the cells were washed with PBS, fixed with methanol for $10 \mathrm{~min}$ and washed with PBS again. The chamber was stained with crystal violet for $20 \mathrm{~min}$ and then washed with PBS. The cells on the upper transwell chamber surface that failed to perforate the chamber were gently wiped off with a cotton swab. The perforating cells on the lower chamber surface were observed and pictured under an inverted microscope (Nikon, Japan). Five fields of view were randomly selected to count the number of perforating cells; the average count was calculated.

\section{Cell invasion assay}

$50 \mu$ l of diluted Matrigel (BD Biosciences, Bedford MA) was added to the upper chamber of the transwell. The chamber was cultured overnight at $37^{\circ} \mathrm{C}$ in a $5 \% \mathrm{CO}_{2}$ incubator to reconstitute the basement membrane. On the next day, serum-starved LV-NC and LV-CXCR7-1 cells were resuspended at cell density of $5 \times 10^{5}$ cells/ml in medium containing $1 \%$ FBS; $200 \mu$ of the cell suspension was added to the upper chamber of a 24-well transwell coated with Matrigel, and $600 \mu \mathrm{l}$ of medium containing $10 \%$ FBS with or without SDF-1 $(100 \mathrm{ng} / \mathrm{ml})$ was added to the lower chamber. The culture plates were transferred to an incubator for $48 \mathrm{~h}$. Thereafter, the procedures were the same as those described for the cell migration assay. The number of perforating cells among the four groups was compared.

\section{Statistical analysis}

SPSS version 13.0 (SPSS Inc., Chicago, IL) was used for statistical analysis. The data were shown as the mean \pm standard deviation (SD). One-way ANOVA and LSD-t test were employed to compare betweengroup comparisons. It was considered to be significant different when $P<0.05$.

\section{Results}

\section{CXCR7 knockdown in SGC-7901 gastric cancer cells}


CXCR7 was knockdown using the shRNA-expressing LV. LV-NC did not change the expression of CXCR7. The mRNA and protein expression of CXCR7 were significantly lower in LV-CXCR7-1 transfected cells compared with Control or LV-NC or LV-CXCR7-2 transfected cells (all $P<0.01$ ). (Figure 1). Therefore, LVCXCR7-I cells were used in the following experiments.

\section{SDF-1/CXCR7 promoted the migration and invasion abilities of gastric cancer cells}

Transwell migration and invasion assay were used to determine the migration and invasion abilities. The number of perforating cells among the four groups was compared. The results showed that compared with that in the LV-NC group, the number of perforating cells in the LV-NC+SDF-1 group was markedly higher $(P<0.01)$, and the number in the LV-CXCR7-1 group was lower. Meanwhile, the number of perforating cells in the LV-CXCR7-1+SDF-1 group was significantly lower compared with that in the LVNC+SDF-1 group $(P<0.01)$. In addition, transwell invasion assay showed that SDF-1 markedly enhanced the invasion abilities of SGC-7901 gastric cancer cells and CXCR7 knockdown inhibited these effects (Figure 2).

\section{SDF-1/CXCR7 promoted the EMT of gastric cancer cells}

To explore the effects of SDF-1/CXCR7 on the EMT of gastric cancer cells, RT-qPCR and western blot were performed to detect the mRNA and protein expression of EMT markers including E-cadherin, N-cadherin, vimentin and Snail. The results are shown in Figure 3. Compared with that in the LV-NC group, the Ecadherin expression in the LV-NC+SDF-1 group were significantly lower $(P<0.05$ and $P<0.01)$, and the expression of $\mathrm{N}$-cadherin, vimentin and Snail were significantly higher (all $P<0.01$ ); compared with those in the LV-NC group, E-cadherin expression in the LV-CXCR7-1 group were higher $(P<0.05)$, while the expression of $\mathrm{N}$-cadherin and vimentin were lower (all $P<0.05$ ); And Snail expression was not significantly different between the two groups. Compared with those in the LV-NC+SDF-1 group, Ecadherin expression in the LV-CXCR7-1+SDF-1 group were significantly higher $(P<0.01)$, while the expression of $\mathrm{N}$-cadherin, vimentin, and Snail were significantly lower (all $P<0.01$ ). These results indicated that SDF-1 could promote the EMT process in gastric cancer cells through CXCR7.

\section{SDF-1/CXCR7 increased the expression of matrix metalloproteinase 2 (MMP-2), MMP-9 and VEGF in gastric cancer cells}

To explore the mechanism of SDF-1/CXCR7 inhibiting migration and invasion, we also detected the expression of MMP-2, MMP-9 and VEGF. As shown in Figure 4, compared with those in the LV-NC group, the expression of MMP-2, MMP-9 and VEGF in the LV-NC+SDF-1 group were significantly higher (all $P<0.01)$; MMP-2 expression in the LV-CXCR7-1 group were similar; and the expression of MMP-9 and VEGF were lower $(P<0.05$ and $P<0.01)$. Compared with those in the LV-NC+SDF-1 group, the expression of MMP-2, MMP-9 and VEGF in the LV-CXCR7-1+SDF-1 group were significantly lower (all $P<0.01$ ). These results indicated that SDF-1 could promote the expression of MMP-2, MMP-9 and VEGF in gastric cancer cells through CXCR7. 


\section{SDF-1/CXCR7 activated the Akt pathway in gastric cancer cells}

Akt is a key protein in the PI3K/Akt signaling pathway and the activation of this signaling pathway is closely related to cell metastasis, angiogenesis and EMT, so we detected the expression of p-Akt and t-Akt in gastric cancer cells. As shown in Figure 5, the level of p-Akt increased significantly at 5 min after SDF-1 stimulation $(P<0.01)$, and the difference in p-Akt levels was not significant among $5 \mathrm{~min}, 10 \mathrm{~min}$ and 30 min. In addition, SDF-1 stimulation did not affect t-Akt levels. Therefore, gastric cells were treated with SDF-1 for $10 \mathrm{~min}$ in the following experiments. Compared with that in the LV-NC group, the p-Akt level in the LV-NC+SDF-1 group was significantly higher $(P<0.01)$, while the $\mathrm{p}-A k t$ level in the LV-CXCR7-1 group was statically similar. Compared with that in the LV-NC+SDF-1 group, the p-Akt level in the LV-CXCR7$1+$ SDF-1 group was significantly lower $(P<0.01)$. Additionally, CXCR7 knockdown did not affect t-Akt levels. These results suggest that SDF-1 activated the Akt signaling pathway in gastric cancer cells through CXCR7.

\section{Discussion}

Gastric cancer metastasis greatly increases the difficulty of treatment and it becomes the main reason for gastric cancer deaths. This process involves multiple steps, including the mesenchymal transformation of tumor cells, the separation and shedding of tumor cells, enhanced adhesion with the extracellular matrix, the degradation of extracellular matrix, migration and invasion, enhanced angiogenesis and tumor cell proliferation activity, resulting in the production of new metastatic lesions. It has been shown that CXCR7 is involved in tumor metastasis. For example, CXCR7 expression in osteosarcoma tissue was associated with distant tumor metastasis [18]. High CXCR7 expression in papillary thyroid carcinoma correlates with lymph node metastasis and distant metastasis [19]. In animal experiments, it was found that CXCR7 increased lung metastasis of colon cancer and liver cancer $[7,20]$ and increased liver metastasis of pancreatic cancer [9]. A number of in vitro studies have also found that SDF-1/CXCR7 enhanced the migration and invasion abilities of cancer cells, including liver cancer [6], pancreatic cancer [9], kidney cancer [11], breast cancer [12], ovarian cancer [13], glioma [17], and papillary thyroid carcinoma [21]. In our current study, we found that SDF-1/CXCR7 could promote the migration and invasion ability of SGC-7901 gastric cancer cells by using a transwell chamber migration and invasion model in vitro.

The mechanism of tumor invasion and metastasis is very complex, and EMT has been a research hotspot in recent years. More than $90 \%$ of human malignant tumors are epithelial tumors. When EMT occurs in tumor cells, it is manifested as the reduction and loss of epithelial characteristics and the acquisition of mesenchymal characteristics, ultimately enhancing the mobility and invasion ability of cells and promoting the invasion and metastasis of tumors. E-cadherin expression is downregulated in EMT, perhaps the most important change in the process, while the expression of the mesenchymal markers $\mathrm{N}$-cadherin and vimentin is upregulated in EMT. The Snail transcription factor is a regulatory molecule of EMT that can downregulate E-cadherin expression by directly binding to its promoter $[22,23]$. Studies have shown that CXCR7 promotes EMT and migration and/or invasion abilities in bladder cancer [16], lung cancer [24] and breast cancer [25]. Li et al. found that in breast cancer the expression of the 
transcription factor Snail in a CXCR7 gene silencing group after SDF-1 stimulation was reduced[25]. Hu et al. found that the SDF-1/CXCR4 axis also significantly promoted the invasion ability of tumor cells, reduced E-cadherin expression and increased the expression of N-cadherin and vimentin, while CXCR4 also promoted the expression of Wnt pathway-related genes and target genes; blocking the Wnt pathway downregulated E-cadherin, upregulated $\mathrm{N}$-cadherin and Snail, and increased the invasive ability of cells, suggesting that SDF-1/CXCR4 promotes colorectal cancer progression and EMT processes through the Wnt/ $\beta$-catenin pathway [26]. Our study showed that after the administration of SDF-1, E-cadherin expression was significantly decreased and the expression of $\mathrm{N}$-cadherin, vimentin, and Snail were significantly increased. In contrast, CXCR7 knockdown increased E-cadherin levels and decreased Ncadherin, vimentin and Snail levels. These results suggest that SDF-1/CXCR7 promoted EMT in gastric cancer and that the occurrence of EMT was related to the activation of Snail. The promotion of EMT by SDF-1/CXCR7 may be one of the mechanisms by which SDF-1/CXCR7 promotes the migration and invasion ability of gastric cancer cells.

In humans, matrix metalloproteinases especially MMP-2 and MMP-9 are the main gelatinases. They play key roles in the hydrolysis of the extracellular matrix and basement membrane, thus promoting tumor invasion and metastasis [27]. Li et al. found that SDF-1/CXCR7 promoted breast cancer cell invasion through increasing the expression of MMP-2 and MMP-9 [25]. Yu et al. found that SDF-1/CXCR7 induced the expression of MMP-9 through the 38 MAPK signaling pathway in ovarian cancer, thereby promoting the invasive ability of cancer cells [13]. Our current study showed that SDF-1 could promote the expression of MMP-2 and MMP-9 in gastric cancer cells through CXCR7, which may be another one of the mechanisms by which SDF-1/CXCR7 promotes the migration and invasion ability of gastric cancer cells. VEGF acts on endothelial cells by binding to specific receptors, thereby inducing the growth and proliferation of endothelial cells [28], inhibiting apoptosis, promoting vascular construction, improving vascular permeability and promoting endothelial cell migration [29]. Studies have shown that SDF-1 increases VEGF mRNA expression in human umbilical vein endothelial cells and promotes VEGF- induced DNA synthesis [30]. CXCR7 promotes VEGFA accumulation in tumor tissues and serum of nude mice with hepatocellular carcinoma xenografts. In addition, antibody arrays and enzyme-linked immunosorbent assays were used to validate the promoting effect of CXCR7 on VEGFA in vitro [7]. Zheng et al. found that CXCR7 induces the formation of a lumen by human umbilical vein endothelial cells in vitro and promotes hepatocellular carcinoma angiogenesis in vivo. In addition, SDF-1/CXCR7 induces VEGF secretion from hepatoma cells, and VEGF positively regulates CXCR7 expression [8]. The induction of VEGF by SDF$1 / C X C R 7$ has also been confirmed in prostate cancer and bladder cancer $[14,16]$. Our current study showed that SDF-1 promoted VEGF expression in gastric cancer cells through CXCR7, suggesting that SDF-1/CXCR7 may promote the angiogenesis of gastric cancer.

Although it has been confirmed that CXCR7 does not activate the G protein-dependent signaling pathway, it can recruit $\beta$-arrestin for signal transduction [31-34]. Our previous study showed that SDF-1 activated ERK $1 / 2$ and p38 signaling pathway in gastric cancer cells. Akt, also known as protein kinase $B$, is a key protein in the PI3K/Akt pathway and its activation is closely related to cell apoptosis, cell proliferation, cell cycle regulation, cell invasion and metastasis, angiogenesis and the promotion of telomerase activity 
in human malignancies [35, 36]. Wang et al. demonstrated that SDF-1/CXCR7 promoted Akt phosphorylation in prostate cancer [14]. CXCR7 can also increase the p-Akt level in osteosarcoma [18], bladder cancer [16], thyroid cancer [37], and multiple myeloma [38]. Our study also showed that SDF1/CXCR7 increased the p-Akt level in gastric cancer cells, indicating the activation effect of SDF-1/CXCR7 on Akt signal transduction. We speculate that the activation effect of SDF-1/CXCR7 on Akt may be related to the biological characteristics of promoting metastasis and EMT; however, the specific mechanism needs to be further elucidated.

\section{Conclusions}

In summary, this study showed that SDF-1/CXCR7 enhanced the migration and invasion ability of the gastric cancer cell, increased expression of MMP-2, MMP-9 and VEGF, promoted EMT and activated the Akt pathway. On the basis of our previous studies, these results further elucidate the mechanisms of SDF$1 / C X C R 7$ in promoting gastric cancer progression and provide experimental basis for molecular therapy. Because CXCR7 and CXCR4 are co-expressed in gastric cancer cells and can form homodimers and heterodimers. These results also offer a basis of studying the role of SDF-1/CXCR4/CXCR7 axis in gastric cancer progress. However, we only used SGC-7901 cells to study the mechanisms of SDF-1/CXCR7 on gastric cancer cell metastasis and only focused on the migration, invasion and EMT. In future, we will further study the underlying signal transduction and expression regulation mechanisms in future.

\section{Declarations}

\section{Funding:}

This study was supported by the Science and Technology Benefit the People Program of Shaanxi (No. 2017HM-01), Foundation of the Second Affiliated Hospital of Xi'an Jiaotong University [No. YJ (QN) 201514].

\section{Conflicts of interest/Competing interests:}

There are no conflicts of interest.

\section{Availability of data and material:}

All data are available within the article, or available from the authors upon request.

\section{Code availability (software application or custom code):}

Not applicable.

\section{Authors' contributions :}


Haitao Shi developed the conception and design of the study. Ameng Shi and Ting Wang conducted the experiments and wrote the manuscript. Ameng Shi, Ting Wang and Miao Jia performed the data analysis. Lei Dong and Haitao Shi revised the manuscript.

Additional declarations for articles in life science journals that report the results of studies involving humans and/or animals:

Not applicable.

\section{Ethics approval:}

This article does not contain any studies with human participants or animals performed by any of the authors.

\section{Consent to participate (include appropriate statements):}

All authors participated in this manuscript.

\section{Consent for publication (include appropriate statements):}

All authors approved the final manuscript for publication.

\section{References}

1. Bray F, Ferlay J, Soerjomataram I, Siegel RL, Torre LA, Jemal A (2018) Global cancer statistics 2018: GLOBOCAN estimates of incidence and mortality worldwide for 36 cancers in 185 countries. CA Cancer J Clin 68(6):394-424. http://doi.org/10.3322/caac.21492

2. Irino T, Matsuda S, Wada N, Kawakubo H, Kitagawa Y (2021) Essential updates 2019/2020: Perioperative and surgical management of gastric cancer. Annals of Gastroenterological Surgery 5(2):162-172. https://doi.org/10.1002/ags3.12438

3. Xue LJ, Mao XB, Ren LL, Chu XY (2017) Inhibition of CXCL12/CXCR4 axis as a potential targeted therapy of advanced gastric carcinoma. Cancer Med 6(6):1424-1436. http://doi.org/10.1002/cam4.1085

4. Graham GJ, Locati M, Mantovani A, Rot A, Thelen M (2012) The biochemistry and biology of the atypical chemokine receptors. Immunol Lett 145(1-2):30-38. http://doi.org/10.1016/j.imlet.2012.04.004

5. Shi A, Shi H, Dong L, Xu S, Jia M, Guo X, Wang T (2017) CXCR7 as a chemokine receptor for SDF-1 promotes gastric cancer progression via MAPK pathways. Scand J Gastroenterol 52(6-7):745-753. http://doi.org/10.1080/00365521.2017.1300681

6. Neve Polimeno M, lerano C, D'Alterio C, Simona Losito N, Napolitano M, Portella L, Scognamiglio G, Tatangelo F, Maria Trotta A, Curley S (2015) CXCR4 expression affects overall survival of HCC 
patients whereas CXCR7 expression does not. Cell Mol Immunol 12(4):474-482.

http://doi.org/10.1038/cmi.2014.102

7. Lin L, Han MM, Wang F, Xu LL, Yu HX, Yang PY (2014) CXCR7 stimulates MAPK signaling to regulate hepatocellular carcinoma progression. Cell Death Dis 5:e1488.

http://doi.org/10.1038/cddis.2014.392

8. Zheng K, Li HY, Su XL, Wang XY, Tian T, Li F, Ren GS (2010) Chemokine receptor CXCR7 regulates the invasion, angiogenesis and tumor growth of human hepatocellular carcinoma cells. J Exp Clin Cancer Res 29(1):31-31. http://doi.org/10.1186/1756-9966-29-31

9. Guo JC, Li J, Zhou L, Yang JY, Zhang ZG, Liang ZY, Zhou WX, You L, Zhang TP, Zhao YP (2016) CXCL12-CXCR7 axis contributes to the invasive phenotype of pancreatic cancer. Oncotarget 7(38):62006-62018. http://doi.org/10.18632/oncotarget.11330

10. Xu H, Wu Q, Dang S, Jin M, Xu J, Cheng Y, Pan M, Wu Y, Zhang C, Zhang Y (2011) Alteration of CXCR7 expression mediated by TLR4 promotes tumor cell proliferation and migration in human colorectal carcinoma. PLoS One 6(12):e27399. http://doi.org/10.1371/journal.pone.0027399

11. Ierano C, Santagata S, Napolitano M, Guardia F, Grimaldi A, Antignani E, Botti G, Consales C, Riccio A, Nanayakkara M (2014) CXCR4 and CXCR7 transduce through mTOR in human renal cancer cells. Cell Death Dis 5:e1310. http://doi.org/10.1038/cddis.2014.269

12. Wani N, Nasser MW, Ahirwar DK, Zhao H, Miao Z, Shilo K, Ganju RK (2014) C-X-C motif chemokine 12/C-X-C chemokine receptor type 7 signaling regulates breast cancer growth and metastasis by modulating the tumor microenvironment. Breast Cancer Res 16(3):R54. http://doi.org/10.1186/bcr3665

13. Yu Y, Li H, Xue B, Jiang X, Huang K, Ge J, Zhang H, Chen B (2014) SDF-1/CXCR7 axis enhances ovarian cancer cell invasion by MMP-9 expression through p38 MAPK pathway. DNA Cell Biol 33(8):543-549. http://doi.org/10.1089/dna.2013.2289

14. Wang J, Shiozawa Y, Wang J, Wang Y, Jung Y, Pienta KJ, Mehra R, Loberg R, Taichman RS (2008) The role of CXCR7/RDC1 as a chemokine receptor for CXCL12/SDF-1 in prostate cancer. J Biol Chem 283(7):4283-4294. http://doi.org/10.1074/jbc.M707465200

15. Yang J, Tang H, Huang J, An H (2018) Upregulation of CXCR7 Is Associated with Poor Prognosis of Prostate Cancer. Med Sci Monit 24:5185-5191. http://doi.org/10.12659/MSM.906180

16. Hao M, Zheng J, Hou K, Wang J, Chen X, Lu X, Bo J, Xu C, Shen K, Wang J (2012) Role of chemokine receptor CXCR7 in bladder cancer progression. Biochem Pharmacol 84(2):204-214. http://doi.org/10.1016/j.bcp.2012.04.007

17. Liu C, Pham K, Luo D, Reynolds BA, Hothi P, Foltz G, Harrison JK (2013) Expression and functional heterogeneity of chemokine receptors CXCR4 and CXCR7 in primary patient-derived glioblastoma cells. PLoS One 8(3):e59750. http://doi.org/10.1371/journal.pone.0059750

18. Zhang Y, Yang CQ, Gao Y, Wang C, Zhang CL, Zhou XH (2014) Knockdown of CXCR7 inhibits proliferation and invasion of osteosarcoma cells through inhibition of the PI3K/Akt and beta-arrestin pathways. Oncol Rep 32(3):965-972. http://doi.org/10.3892/or.2014.3290 
19. Dang S, Peng Y, Ye L, Wang Y, Qian Z, Chen Y, Wang X, Lin Y, Zhang X, Sun X (2013) Stimulation of TLR4 by LMW-HA induces metastasis in human papillary thyroid carcinoma through CXCR7. Clin Dev Immunol 2013:712561. http://doi.org/10.1155/2013/712561

20. Guillemot E, Karimdjee-Soilihi B, Pradelli E, Benchetrit M, Goguet-Surmenian E, Millet MA, Larbret F, Michiels JF, Birnbaum D, Alemanno P (2012) CXCR7 receptors facilitate the progression of colon carcinoma within lung not within liver. Br J Cancer 107(12):1944-1949. http://doi.org/10.1038/bjc.2012.503

21. Liu Z, Yang L, Teng X, Zhang H, Guan H (2014) The involvement of CXCR7 in modulating the progression of papillary thyroid carcinoma. J Surg Res 191(2):379-388. http://doi.org/10.1016/j.jss.2014.04.016

22. Chou YS, Yang MH (2015) Epithelial-mesenchymal transition-related factors in solid tumor and hematological malignancy. J Chin Med Assoc 78(8):438-445. http://doi.org/10.1016/j.jcma.2015.05.002

23. Ribatti D , Tamma R , Annese T (2020) Epithelial-Mesenchymal Transition in Cancer: A Historical Overview. Translational oncology 13(6):100773 http://doi.org/10.1016/j.tranon.2020.100773

24. Wu YC, Tang SJ, Sun GH, Sun KH (2016) CXCR7 mediates TGFbeta1-promoted EMT and tumorinitiating features in lung cancer. Oncogene 35(16):2123-2132. http://doi.org/10.1038/onc.2015.274

25. Li JT, Jia LT, Liu NN, Zhu XS, Liu QQ, Wang XL, Yu F, Liu YL, Yang AG, Gao CF (2015) MiRNA-101 inhibits breast cancer growth and metastasis by targeting $\mathrm{CX}$ chemokine receptor 7. Oncotarget 6(31):30818-30830. http://doi.org/10.18632/oncotarget.5067

26. Hu TH, Yao Y, Yu S, Han LL, Wang WJ, Guo H, Tian T, Ruan ZP, Kang XM, Wang J (2016) SDF1/CXCR4 promotes epithelial-mesenchymal transition and progression of colorectal cancer by activation of the Wnt/beta-catenin signaling pathway. Cancer Lett 354(2):417-426. http://doi.org/10.1016/j.canlet.2014.08.012

27. Shimoda M , Ohtsuka T , Okada Y (2020) Stromal metalloproteinases Crucial contributors to the tumor microenvironment. Pathology International 71(1):1-14. http://doi.org/10.1111/pin.13033

28. Oguntade AS, Al-Amodi F, Alrumayh A, Alobaida M, Bwalya M (2021) Anti-angiogenesis in cancer therapeutics: the magic bullet. Journal of the Egyptian National Cancer Institute 33(1):15.http://doi.org/10.1186/s43046-021-00072-6

29. Nadine M , Roula T, Nada A, Joelle EH, Riad S, Ray H, Issam R, George H, Rajesh M (2017) Gastrointestinal cancer cells tr eatment with bevacizumab activates a VEGF autoregulatory mechanism involving telomerase catalytic subunit hTERT via PI3K-AKT, HIF-1a and VEGF receptors. Plos One 12(6):e0179202. http://doi.org/10.1371/journal.pone.0179202

30. Neuhaus T, Stier S, Totzke G, Gruenewald E, Fronhoffs S, Sachinidis A, Vetter H, Ko YD (2003) Stromal cell-derived factor 1alpha (SDF-1alpha) induces gene-expression of early growth response-1 (Egr-1) and VEGF in human arterial endothelial cells and enhances VEGF induced cell proliferation. Cell Proliferation 36(2):75-86. http://doi.org/10.1046/j.1365-2184.2003.00262.x 
31. Levoye A, Balabanian K, Baleux F, Bachelerie F, Lagane B (2009) CXCR7 heterodimerizes with CXCR4 and regulates CXCL12-mediated G protein signaling. Blood 113(24):6085-6093. http://doi.org/10.1182/blood-2008-12-196618

32. Decaillot FM, Kazmi MA, Lin Y, Ray-Saha S, Sakmar TP, Sachdev P (2011) CXCR7/CXCR4 heterodimer constitutively recruits beta-arrestin to enhance cell migration. J Biol Chem 286(37):32188-32197. http://doi.org/10.1074/jbc.M111.277038

33. Coggins NL, Trakimas D, Chang SL, Ehrlich A, Ray P, Luker KE, Linderman JJ, Luker GD (2014) CXCR7 controls competition for recruitment of beta-arrestin 2 in cells expressing both CXCR4 and CXCR7. PLoS One 9(6):e98328. http://doi.org/10.1371/journal.pone.0098328

34. Rajagopal S, Kim J, Ahn S, Craig S, Lam CM, Gerard NP, Gerard C, Lefkowitz RJ (2010) Beta-arrestinbut not G protein-mediated signaling by the "decoy" receptor CXCR7. Proc Natl Acad Sci USA 107(2):628-632. http://doi.org 10.1073/pnas.0912852107

35. Cao F, Zhang C, Han W, Gao XJ, Ma J, Hu YW, Gu X, Ding HZ, Zhu LX, Liu Q (2017) p-Akt as a potential poor prognostic factor for gastric cancer: a systematic review and meta-analysis. Oncotarget 8(35):59878-59888. http://doi.org/10.18632/oncotarget.17001

36. Khorasani A, Pourbagheri-Sigaroodi A, Pirsalehi A, Safaroghli-Azar A, Bashash D (2021) The $\mathrm{pi} 3 \mathrm{k} / \mathrm{akt} / \mathrm{mtor}$ signaling pathway in gastric cancer; from oncogenic variations to the possibilities for pharmacologic interventions. European Journal of Pharmacology, 898, 173983.

http://doi.org/10.1016/j.ejphar.2021.173983

37. Zhu X, Bai Q, Lu Y, Lu Y, Zhu L, Zhou X, Wu L (2016) Expression and function of CXCL12/CXCR4/CXCR7 in thyroid cancer. Int J Oncol 48(6):2321-2329. http://doi.org/10.3892/ijo.2016.3485

38. Azab AK, Sahin I, Moschetta M, Mishima Y, Burwick N, Zimmermann J, Romagnoli B, Patel K, Chevalier E, Roccaro AM (2014) CXCR7-dependent angiogenic mononuclear cell trafficking regulates tumor progression in multiple myeloma. Blood 124(12):1905-1914.http://doi.org/10.1182/blood2014-02-558742

\section{Tables}

Table 1 Primer sequences for Real-time PCR 


\begin{tabular}{|c|c|c|}
\hline Target genes & \multicolumn{2}{|c|}{ Sequences } \\
\hline \multirow[t]{2}{*}{$\beta$-actin } & Forward & 5'-ATCGTGCGTGACATTAAGGAGAAG-3' \\
\hline & Reverse & 5'-AGGAAGGAAGGCTGGAAGAGTG-3' \\
\hline \multirow[t]{2}{*}{ CXCR7 } & Forward & 5'-CCTGACACCTACTACCTGAAGAC-3' \\
\hline & Reverse & 5'-CACTGGACGCCGAGATGG-3' \\
\hline \multirow[t]{2}{*}{ E-cadherin } & Forward & 5'-ACCAACGATAATCCTCCGAT-3' \\
\hline & Reverse & 5'-TCAGTGTGGTGATTACGACG-3' \\
\hline \multirow[t]{2}{*}{$\mathrm{N}$-cadherin } & Forward & 5'-AATCCTCCAGAGTTTACTGC-3' \\
\hline & Reverse & 5'-TCCTTATCGGTCACAGTTAG-3' \\
\hline \multirow[t]{2}{*}{ Vimentin } & Forward & 5'-GAGAGGAAGCCGAAAACAC-3' \\
\hline & Reverse & 5'-TGCGTTCAAGGTCAAGACG-3' \\
\hline \multirow[t]{2}{*}{ Snail } & Forward & 5'-TTACCTTCCAGCAGCCCTAC-3' \\
\hline & Reverse & 5'-AGAGTCCCAGATGAGCATTG-3' \\
\hline \multirow[t]{2}{*}{ MMP-2 } & Forward & 5'-GACCACAGCCAACTACGATG-3' \\
\hline & Reverse & 5'-ACGGAAGTTCTTGGTGTAGG-3' \\
\hline \multirow[t]{2}{*}{ MMP-9 } & Forward & 5'-ACCTCGAACTTTGACAGCGACA-3' \\
\hline & Reverse & 5'-GATGCCATTCACGTCGTCCTTA-3' \\
\hline \multirow[t]{2}{*}{ VEGF } & Forward & 5'-GGGCAGAATCATCACGAAGT-3' \\
\hline & Reverse & 5'-GAAGATGTCCACCAGGGTCT-3' \\
\hline
\end{tabular}

\section{Figures}


(a)

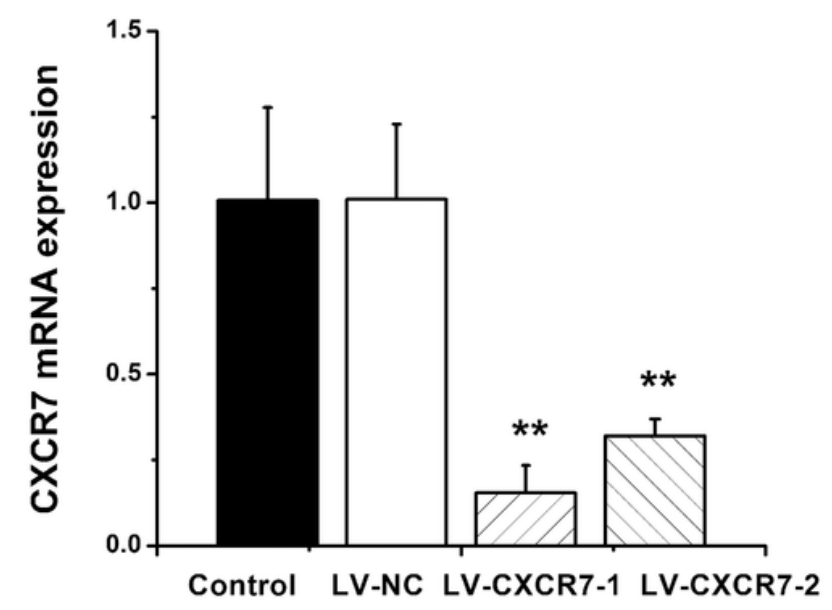

(b)

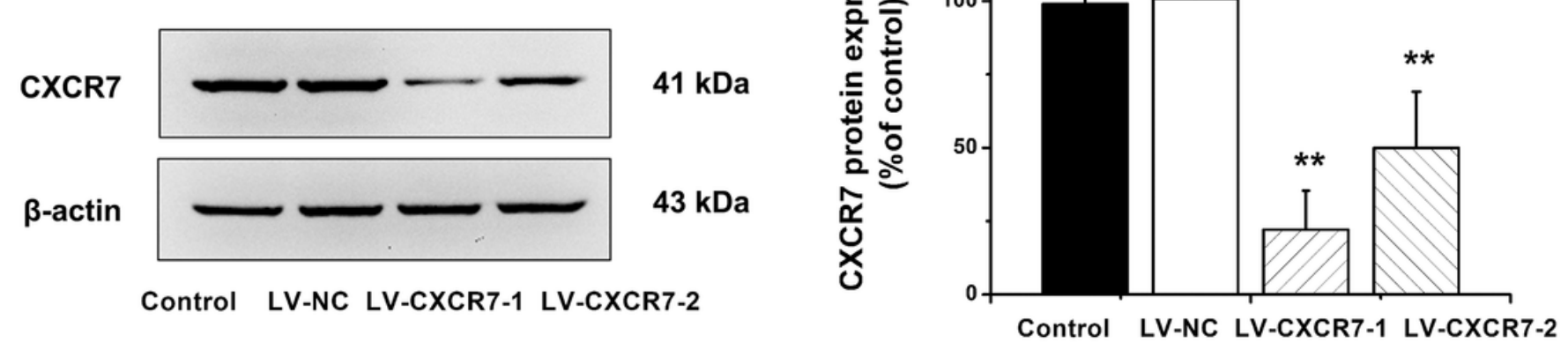

Figure 1

The knockdown of CXCR7 in gastric cancer cells. (a) CXCR7 mRNA expression was detected by RT-qPCR. (b) CXCR7 protein expression was detected by western blot. Data are shown as mean \pm SD. Origin 7.0 software (OriginLab Corporation, USA) was used for creation of histogram. ${ }^{\star \star} \mathrm{P}<0.01$ vs. LV-NC group 
(a)
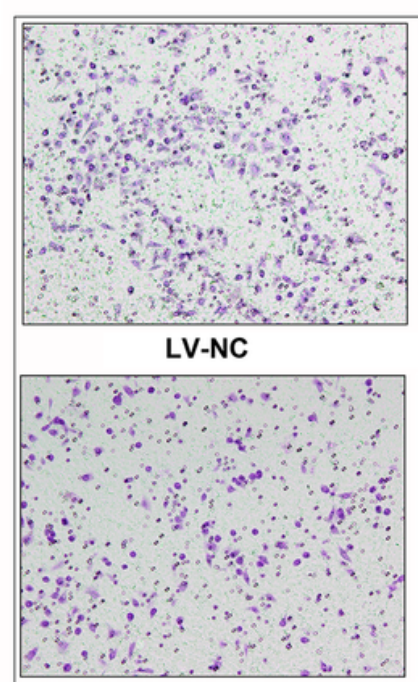

LV-CXCR7-1

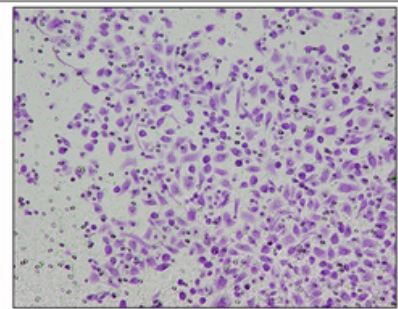

LV-NC+SDF-1

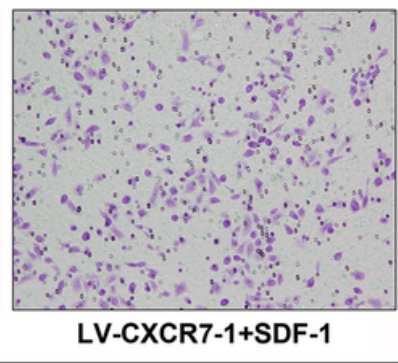

(b)

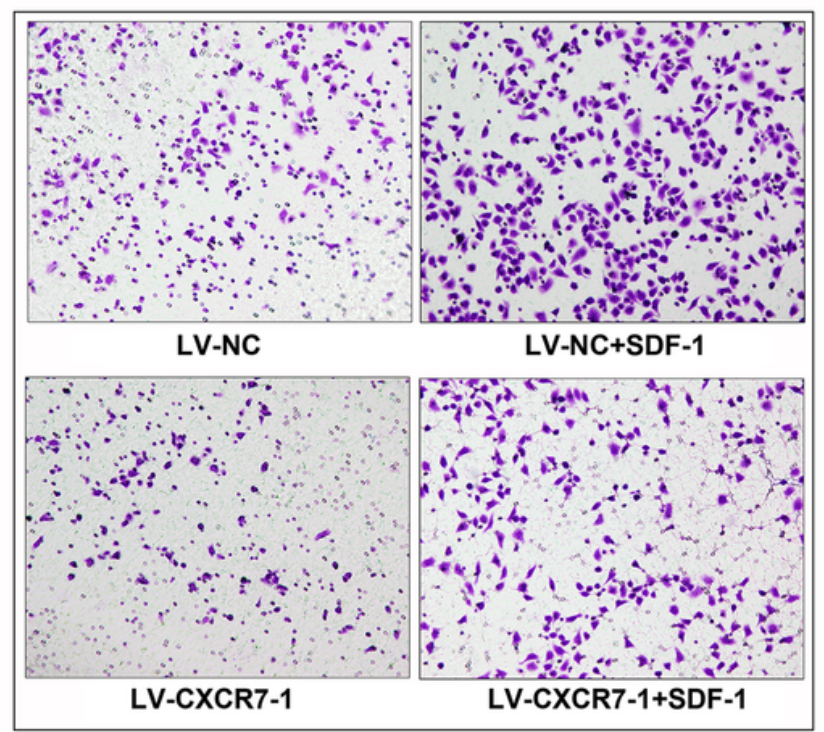

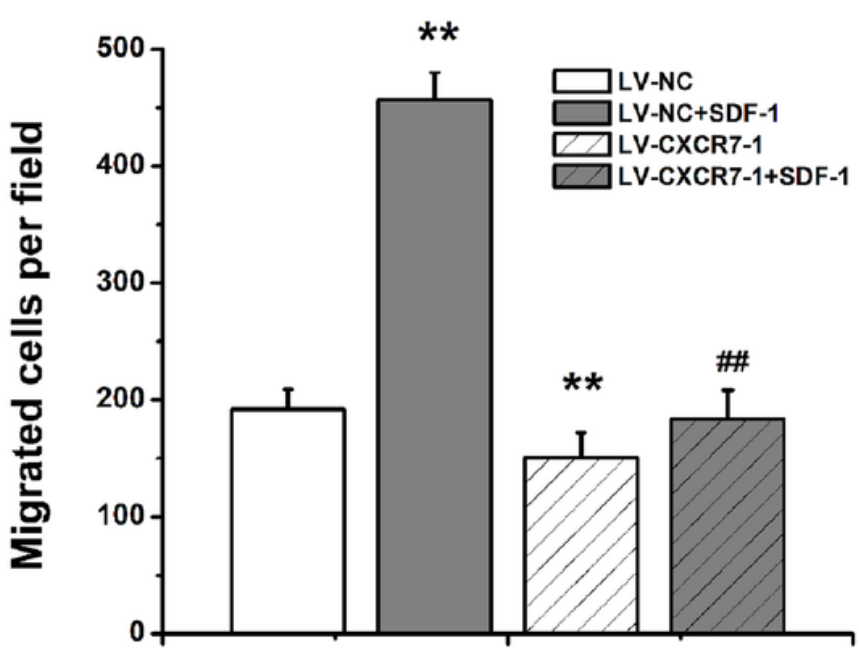

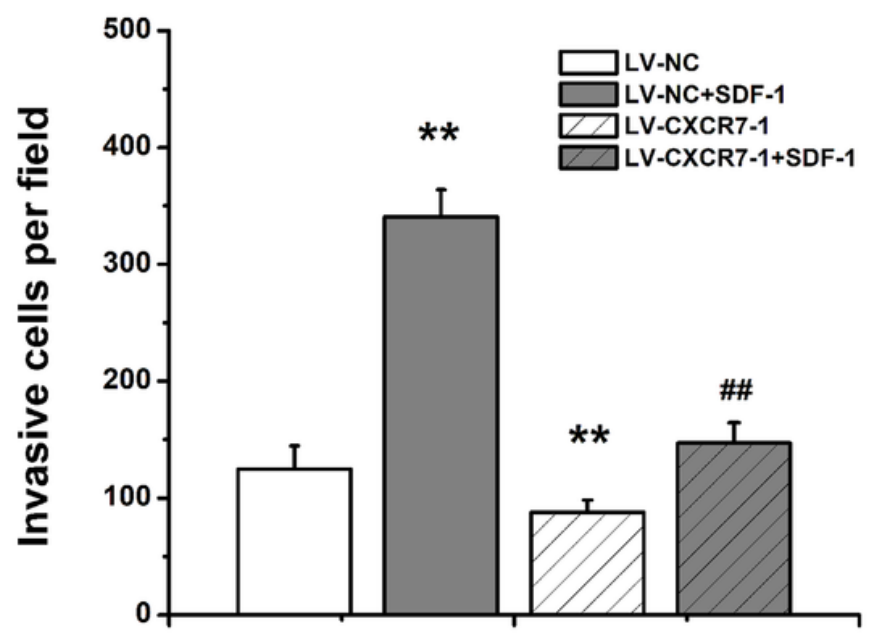

\section{Figure 2}

Effects of SDF-1/CXCR7 on the migration and invasion abilities of gastric cancer cells. (a) Transwell migration assay was used to evaluate the migration abilities. The perforating cells were stained with crystal violet and pictured under an inverted microscope (200x) (Nikon, Japan) . (b) Transwell invasion assay was used to evaluate the invasion abilities. The perforating cells were stained with crystal violet and pictured under an inverted microscope (200x) (Nikon, Japan). Data are shown as mean \pm SD. Origin 7.0 software (OriginLab Corporation, USA) was used for creation of histogram. ${ }^{*} \mathrm{P}<0.01$ vs. LV-NC group; $\# \#$ P $<0.01$ vs. LV-NC+SDF-1 group. 
(a)

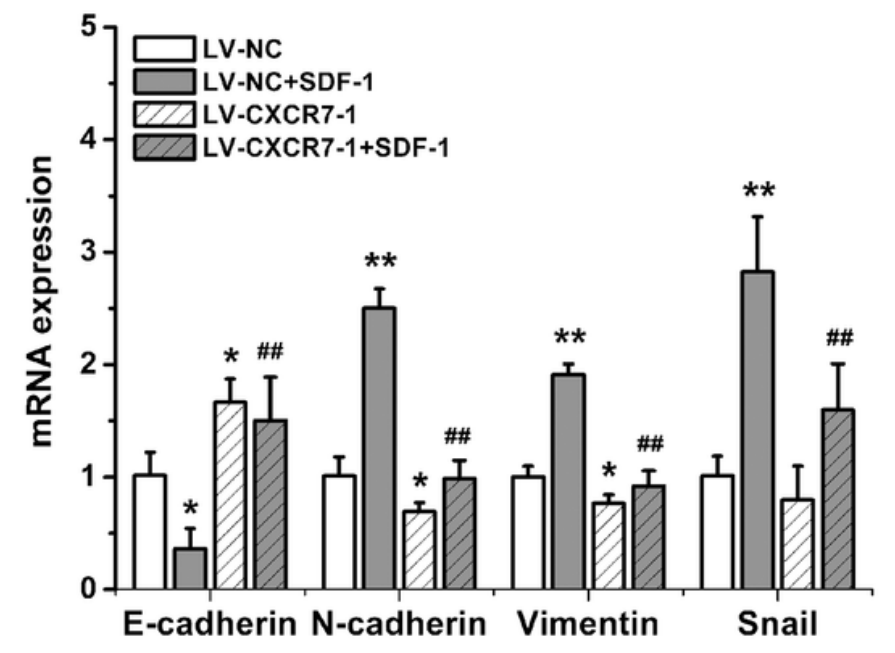

(b)

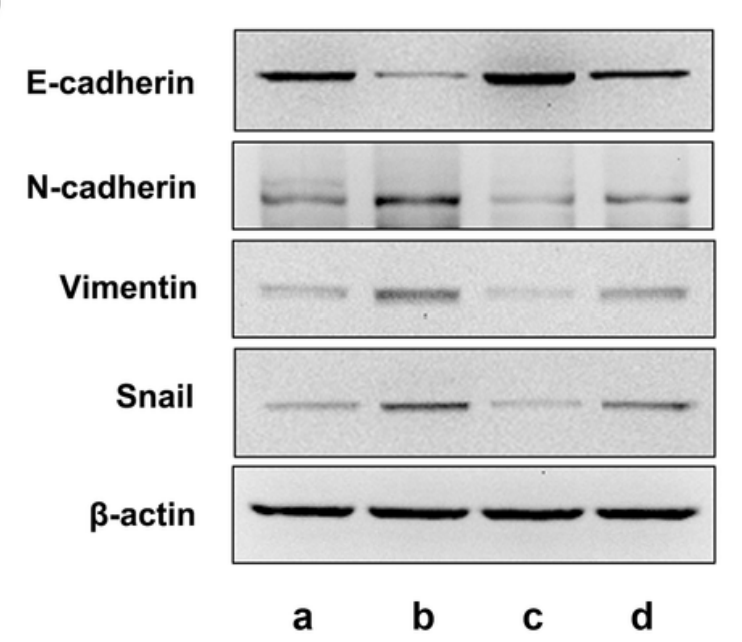

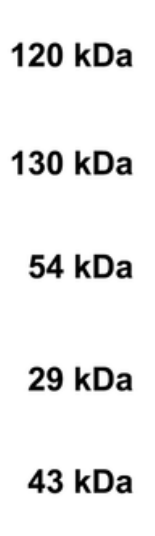

b c c d

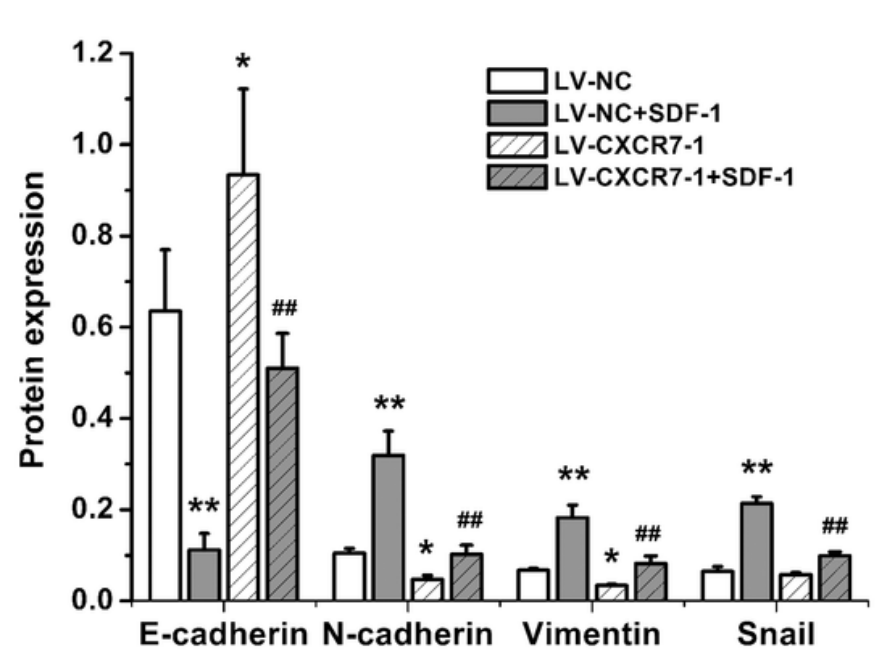

Figure 3

Effects of SDF-1/CXCR7 on EMT in gastric cancer cells. (a) mRNA expression of E-cadherin, N-cadherin, Vimentin and Snail were detected by RT-qPCR. (b) protein expression of E-cadherin, N-cadherin, Vimentin and Snail were detected by western blot. a: LV-NC group; b: LV-NC+SDF-1 group; c: LV-CXCR7-1 group; d: LV-CXCR7-1+SDF-1 group. Data are shown as mean \pm SD. Origin 7.0 software (OriginLab Corporation, USA) was used for creation of histogram. ${ }^{*} P<0.05$, ${ }^{*} P<0.01$ vs. LV-NC group; \#\#P<0.01 vs. LV-NC+SDF-1 group. 
(a)

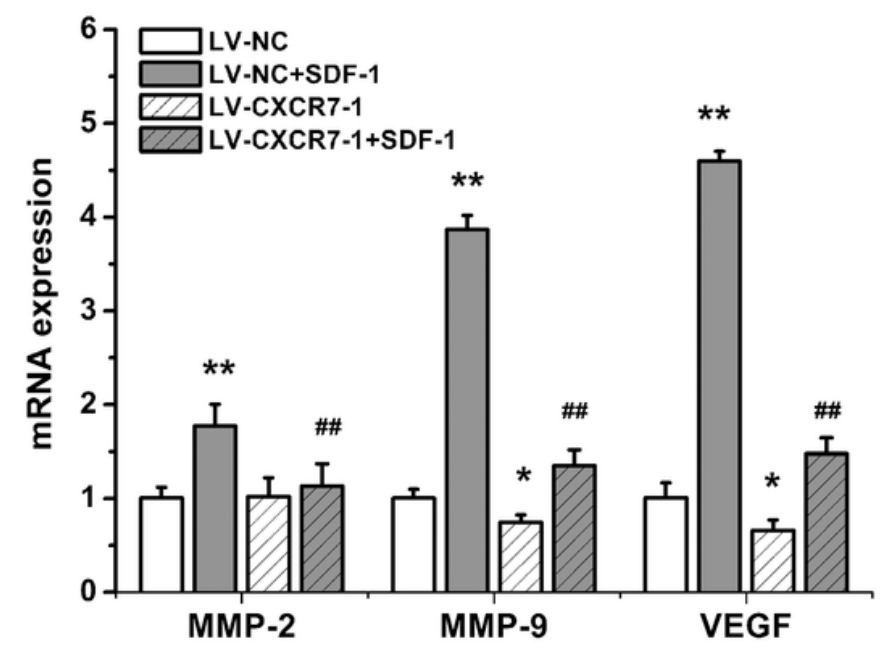

(b)

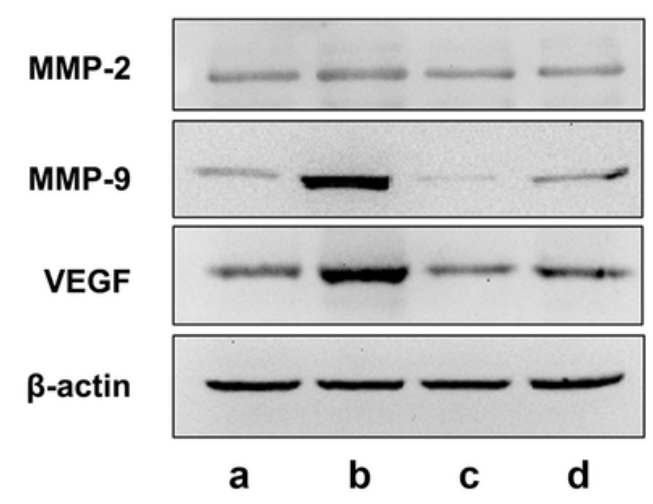

$72 \mathrm{kDa}$

$92 \mathrm{kDa}$

$27 \mathrm{kDa}$

$43 \mathrm{kDa}$

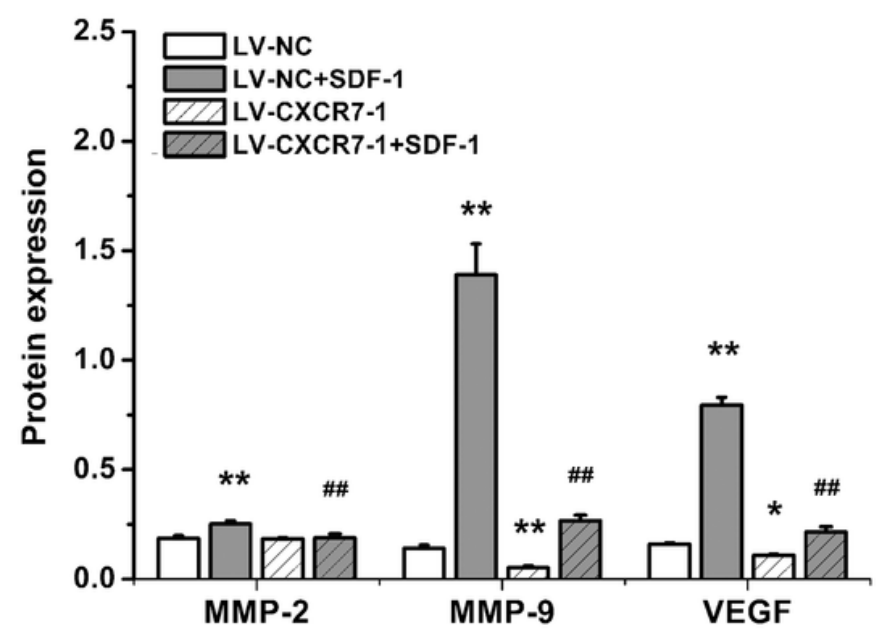

Figure 4

Effects of SDF-1/CXCR7 on the expression of MMP-2, MMP-9 and VEGF in gastric cancer cells. (a) mRNA expression of MMP-2, MMP-9 and VEGF were detected by RT-qPCR. (b) protein expression of MMP-2, MMP-9 and VEGF were detected by western blot. a: LV-NC group; b: LV-NC + SDF-1 group; c: LV-CXCR7-1 group. d: LV-CXCR7-1+SDF-1 group. Data are shown as mean \pm SD. Origin 7.0 software (OriginLab Corporation, USA) was used for creation of histogram. ${ }^{*} \mathrm{P}<0.05$, ${ }^{\star *} \mathrm{P}<0.01$ vs. LV-NC group; \#\#P<0.01 vs. LV-NC+SDF-1 group. 
(a)
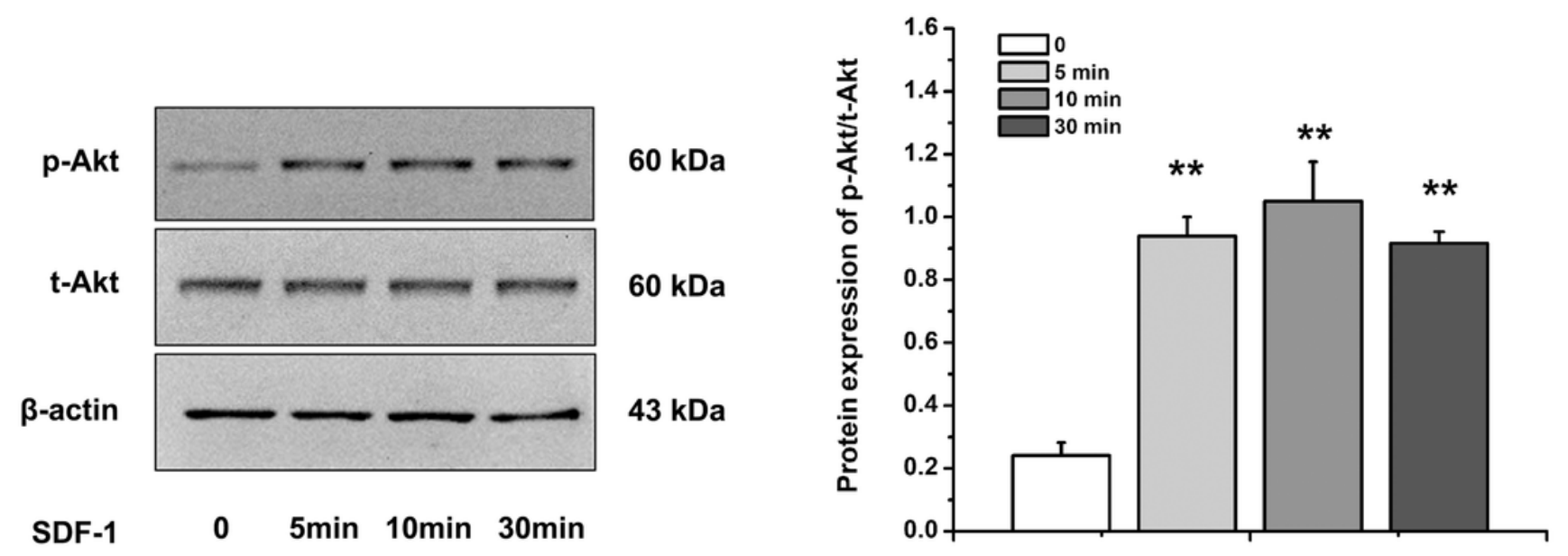

(b)

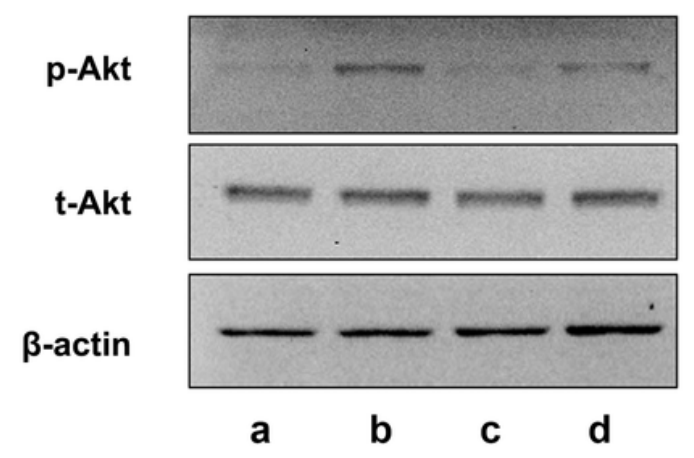

60 kDa

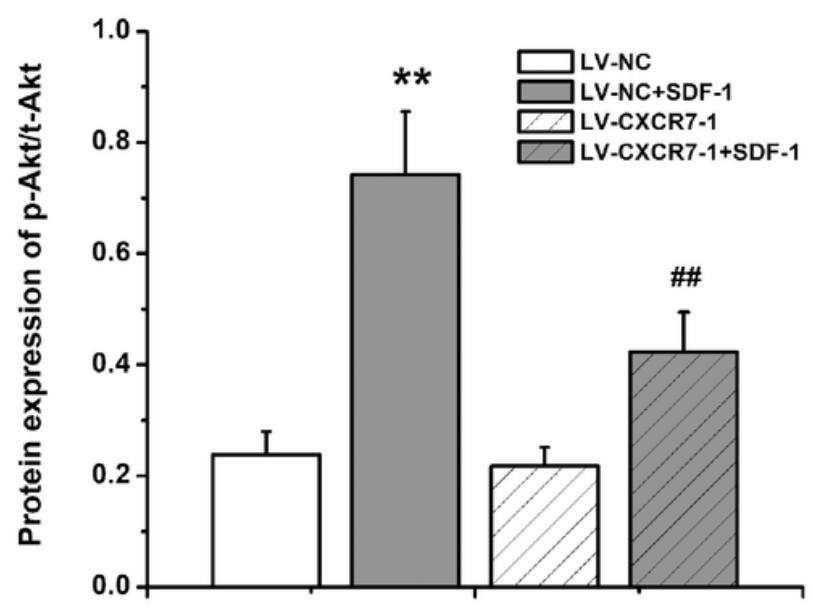

\section{Figure 5}

Effects of SDF-1/CXCR7 on the expression of p-Akt and t-Akt in gastric cancer cells. (a) protein expression of $\mathrm{p}$-Akt and t-Akt after SDF-1 stimulation were detected by western blot. Data are shown as mean $\pm S D$, ${ }^{\star *} P<0.01$ vs. 0 group; (b) expression of $p$-Akt and t-Akt after CXCR7 knockdown were detected by western blot. a: LV-NC group; b: LV-NC+SDF-1 group; c: LV-CXCR7-1 group. d: LV-CXCR7-1+SDF-1 group. Data are shown as mean \pm SD. Origin 7.0 software (OriginLab Corporation, USA) was used for creation of histogram. ${ }^{* *} \mathrm{P}<0.01$ vs. LV-NC group; \#\#P<0.01 vs. LV-NC+SDF-1 group. 